\title{
Ectopic Overexpression of Coiled-Coil Domain Containing 110 Delays G2/M Entry in U2-OS Cells
}

\author{
Sue Nyoung Lee ${ }^{1}$, Kyeong-Man Hong ${ }^{2},{ }^{\dagger}$ Yeon Sun Seong ${ }^{1,3}$, and ${ }^{\dagger}$ Sahng-June Kwak ${ }^{3}$ \\ 'Dept. of Nanobiomedical Science \& BK21 PLUS Research Center for Regenerative Medicine, Dankook University, \\ Cheonan 31116, Korea \\ ${ }^{2}$ Research Institute, National Cancer Center, Goyang 10408, Korea \\ ${ }^{3}$ Dept. of Biochemistry, College of Medicine, Dankook University, Cheonan 31116, Korea
}

Received: April 27, 2020

Revised: May 4, 2020

Accepted: May 19, 2020

${ }^{4}$ Corresponding author

Yeon-Sun Seong

Dept. of Biochemistry,

College of Medicine, Dankook University,

Cheonan 31116, Korea.

Tel: +82-41-550-3875

Fax: +82-41-550-1149

E-mail:seongys@dankook.ac.kr

Sahng-June Kwak

Department of Biochemistry,

College of Medicine, Dankook University,

Cheonan 31116, Korea.

Tel: +82-41-550-3874

Fax: +82-41-550-1149

E-mail:ftpn2100@dankook.ac.kr

Copyright $\odot 2020$ The Korean Society of Developmental Biology.

This is an Open Access article distributed under the terms of the Creative Commons Attribution Non-Commercial License (http://creativecommons.org/licenses/ by-nc/4.0/) which permits unrestricted non-commercial use, distribution, and reproduction in any medium, provided the original work is properly cited.

ORCID

Sue Nyoung Lee

https://orcid.org/0000-0002-4846-212X Kyeong-Man Hong

https://orcid.org/0000-0002-4384-0267 Yeon-Sun Seong

https://orcid.org/0000-0002-4743-8048

Sahng-June Kwak

https://orcid.org/0000-0003-4529-0307

Conflict of interests

The authors declare no potential conflict of interest.

\section{Abstract}

Coiled-coil domain containing 110 (CCDC110, KM-HN-1) is a protein containing C-terminal coiled-coil domain (CCD) which was previously discovered as a member of the human cancer/testis antigen (CTA). In addition, CCDC110 has both nuclear localization signal sequence and the leucine zipper motif. Although the functional role of CCDC110 has yet to be fully identified, the mRNA expression levels of CCDC110 are known to be highly elevated in various cancer types including testis, implying its relevance to cancer pathogenesis. In this study, we first developed several monoclonal antibody (mAb) hybridoma clones targeting CCDC110 and further isolated clone by characterizing for its specificity using immunoblotting and immunoprecipitation approaches with basal parenchymal sperm cells in testis tissue. Next, using these mAbs, we showed that the Tet-inducible overexpression of CCDC110 protein delayed the entry of G2/M phase in U2-OS osteosarcoma cells. Based on these results, we propose that CCDC110 plays a crucial role in cell cycle progression.

Keywords: CCDC110, Cancer/Testis Antigen (CTA), Testis, G2/M, Cell cycle

\section{INTRODUCTION}

Coiled-coil domain containing 110 (CCDC110, KM-HN-1) was initially discovered as a member of the family of human cancer/testis antigens (CTAs), in an attempt to find cancer-specific target proteins for the establishment of cancer treatment modalities and diagnostic methods (Monji et al., 2004). By using RT-PCR analysis of diverse tumor samples and cancer cell lines, the mRNA level of CCDC110 was found to be increased in several cancer types, including tongue cancer, stomach cancer, melanoma, and hepatocellular carcinoma (Monji et al., 2004; Condomines et al., 2007). CTAs are a group of proteins that were reported to exhibit high expression of proteins in specific types of cancers or testicular germ line cells (Chen et al., 1997; Scanlan et al., 2002; Vaughan et al., 2004). Due to their limited protein expression in somatic tissues and escape from immunological surveillance of testicular tissues, they have been considered as a promising target for the establishment of anticancer therapeutics and cancer diagnosis (Chen et al., 1997; Scanlan et al., 2002; Vaughan et al., 2004). To date, more than 270 genes were listed as encoding CTAs in the CTDatabase (http://www.cta.lncc.br) (Chen et al., 1997). CCDC110 contains a C-terminal coiled-coil domain (CCD) in which the $\mathrm{C}$-terminal region 
Acknowledgements

This work was supported by the National

R \& D Program for Cancer Control from

Ministry of Health and Welfare (Grant No.

06201701) to YS Seong. We also appreciate

Prof. JH Ko and YM Lee in the Department of

Pathology for the helpful discussion on the

immunohistochemistry.

Authors' contributions

Conceptualization: Seong YS, Kwak SJ.

Data curation: Lee SN, Hong KM.

Methodology: Lee SN, Hong KM.

Writing - original draft: Lee SN, Seong YS

Writing - review \& editing: Kwak SJ.

Ethics approval

This research was approved from

Dankook University Hospital IRB in 2006 for

human tissue sample immunohistochemistry.

Regarding IACUC approval, as the animal experiment for production of monoclonal antibody was performed between 2004-2005, the approval of IACUC was not essential at that time. of the SMC (structure maintenance of chromatin) structural motif resides. This motif is derived from the SMC protein, which plays an important role in chromosome segregation during anaphase (Cobbe \& Heck, 2000). CCDC110 also includes a bipartite nuclear localization signal (NLS) flanked by a leucine zipper (ZIP) region, in the inner 1/3 region (Boulikas, 1993). The ZIP motif of CCDC110 is consist of 5 leucine-rich repeats (L1, L2, L3, L4, and L5) with intervening six-amino acid residues, and followed by basic amino acids residues like other ZIP motif-containing proteins (Park et al., 2007). The ZIP motif provides a protein-protein interaction domain of a variety of DNA binding proteins (Landschulz et al., 1988).

The role of CCDC110 in cell proliferation and carcinogenesis remains unclear. The NCBI's deposited cDNA microarray data sets (http://www.ncbi.nlm.nih.gov/sites/GDSbrowser) about CCDC110 show evidence that CCDC110 may be related to cell proliferation. The mRNA expression of CCDC110 increases during the period of spermatogenesis, in pachytene spermatocytes and round spermatids (NCBI GEO GDS2390) (Namekawa et al., 2006), in the regenerating liver of mice (NCBI GEO GDS2577) (Otu et al., 2007), and under the overexpression of EGR gene in human umbilical vein endothelial cells (HUVEC) (NCBI GEO GDS2009) (Lucerna et al., 2006). Previously using adenovirus adopted overexpression system, we have identified that ectopically overexpressed of CCDC110 distinctly localized to centrosome and the $\mathrm{C}$-terminal region of the protein seems to be the principal determinant of positioning this protein to the centrosome (Park et al., 2007). The deletion mutant lacking C-terminal CCD displays distinct nuclear localization pattern (Park et al., 2007). Here, we raised monoclonal antibodies against CCDC110 protein and tested it for feasibility to use in immunohistochemical staining (IHC). Ectopic overexpression of CCDC110 using the Tetracycline (Tet) inducible system led to delayed G2/M entry in U2-OS cells.

\section{MATERIALS AND METHODS}

\section{Cell culture}

Mouse hybridoma clone cells were maintained in RPMI 1640 medium (Hyclone Laboratories, Logan, UT, USA) containing the hypoxanthine-thymine (HT) supplement (Sigma-Aldrich, St Louis, MO, USA) and 10\% fetal bovine serum (FBS; Hyclone Laboratories). U2-OS (ATCC, HTB-96), Tet-On U2-OS (Clontech Laboratories, Mountain View, CA, USA), and HEK293T(ATCC, CRL-3216) were maintained in Dulbecco's modified Eagle medium (DMEM, Hyclone Laboratories), supplemented with 10\% FBS and $100 \mathrm{U} / \mathrm{mL}$ of penicillin G plus $100 \mu \mathrm{g} /$ $\mathrm{mL}$ of streptomycin (HyClone Laboratories) at $37^{\circ} \mathrm{C}$ in a $5 \% \mathrm{CO}_{2}$ humidified atmosphere.

\section{Preparations of recombinant CCDC110 proteins}

The coding region of $C C D C 110$ gene corresponding to N-terminal amino acid residue 1-417 of CCDC110 protein (CCDC110N) was amplified by PCR and sub-cloned into the pGEX4T-1 vector (GE Healthcare, Chicago, IL, USA), an expression vector for glutathione $S$-transferase (GST)-fusion protein. Bacterial expression of the GST-tagged CCDC110 protein was induced by the addition of $0.4 \mu \mathrm{M}$ isopropyl-1-thio- $\beta$-D-galactopyranoside (IPTG) and subsequent cultivation for $18 \mathrm{~h}$ at $23^{\circ} \mathrm{C}$. The purification of GST-CCDC110N protein was carried out using glutathione (GSH)-agarose affinity chromatography purification. The purified proteins were used to immunization and enzyme-linked immunosorbent assay (ELISA). 


\section{Productions of monoclonal antibodies}

The production of monoclonal antibody was carried out from 2005 to 2007. $70 \mu \mathrm{g}$ of the purified recombinant GST-CCDC110N protein was emulsified with Complete Freund's Adjuvant (Sigma-Aldrich) and subsequently injected to 6-week-old female Balb/c mice. The boosting immunization was carried out two times for 2-week interval, each immunization with the same amount of purified proteins emulsified with incomplete Freund's adjuvant (Sigma-Aldrich). Generation of GST-CCDC110N-specific antibodies in the serum of immunized mice was tested by both ELISA and immunoblot prior to hybridoma fusion. The hybridoma fusion was performed by standard techniques (Harlow \& Lane, 1988). The immune cells harvested from spleens were fused with SP2/0-Ag14 murine myeloma cells in the presence of polyethylene glycol (PEG) 1500 (Roche, Mannheim, Germany). The obtained hybridoma cell mixtures were transferred into 96well cell culture plates with the method of limiting dilution. The culture soups containing $m A$ bs from the hybridoma cultures were screened by ELISA followed by immunoblotting. Ascites fluid was also produced by peritoneal injection of hybridoma cells into a pristane primed Balb/c mice.

\section{SDS-PAGE and immunoblotting}

SDS-PAGE and Immunoblotting was performed as described previously (Lee, 2012).

For immunoblotting analysis the following commercial primary antibodies were used in this study: anti-Cyclin D1 (\#2922) and anti-Cyclin D3 (\# 2936) were purchased from Cell Signaling Technology (Danvers, MA, USA); anti-Cyclin B1 (H-433), anti-p53 (SC-126), and anti-ERK1 (SC-94) were purchased from Santa Cruz Biotechnology (Santa Cruz, CA, USA); anti-p21 (ab7960) was purchased from Abcam (Cambridge, MA, USA). The primary antibodies were visualized with goat anti-rabbit (SC-2004, Santa Cruz Biotechnology, Santa Cruz) or goat antimouse (SC-2005, Santa Cruz Biotechnology) antibodies conjugated with horseradish peroxidase and an enhanced chemiluminescence (ECL) detection system.

\section{Tet-inducible EGFP-CCDC110 expression system}

The N-terminally enhanced green fluorescence protein (EGFP)-fused full-length open reading frame (ORF) of CCDC110 was PCR amplified and subcloned into pHR CMV*-1 puro SV40 vector and used for generation of lentivirus according to previous report (Soung et al., 2006). The pHR CMV*-1 puro SV40 EGFP-CCDC110 was transfected with pHRCMV-VSVG and pHRCMV-d8.2 dpr into HEK-293T cells and the culture soup containing lentivirus capable of expressing EGFP-CCDC110 and puromycin N-acetyltransferase (PAC) genes. The obtained lentivirus was introduced to Tet-On U2-OS cells and puromycin selection $(2 \mu \mathrm{g} / \mathrm{mL})$ was carried out. With the method of limiting dilution and immunoblotting, the Tet-inducible EGFP-CCDC110 U2OS cell lines were cloned and used for further experiment.

\section{Immunofluorescence staining and confocal microscopy}

U2-OS cells were grown on Poly D-lysine (Sigma-Aldrich)-coated glass coverslips. After wash-out with phosphate-buffered saline (PBS), cells were fixed with 3.7\% formaldehyde solution dissolved in PBS, and then permeabilized with ice cold methanol for 2 min. After blocking with PBS containing 5\% BSA solution, the cells were incubated for $2 \mathrm{~h}$ with each primary antibody. After wash-out the primary Abs, the cells were incubated with Alexa 532-conjugated antimouse IgG (Invitrogen, Carlsbad, CA, USA) was carried out for $2 \mathrm{~h}$ at room temperature. For the visualization of nucleus, Hoechst 33452 (Sigma-Aldrich) was added during the period of $1^{\text {st }}$ washing after secondary antibody application. The stained cells were mounted on glass slides with semi-solidifying mounting solution (Polysciences, Warrington, PA, USA). Confocal fluorescence 
images were obtained by Carl Zeiss LSM 700 Meta microscope system (Carl Zeiss, Thornwood, NY, USA).

\section{Immunohistochemistry staining in human tissue samples}

The IHC experiment using human tissue samples was approved from Dankook University Hospital IRB in 2006. The paraffin embedded tissue blocks of human testis previously obtained from a patient in his 50s who was hospitalized after a car accident were cut into $10-\mu \mathrm{m}$ sections and placed on frosted glass microscope slides. After removal of paraffin with xylene, the tissue sections were dehydrated in a graded alcohol series. For the procedure of antigen retrieval, the tissue sections were heated in a pressured chamber containing $10 \mathrm{mM}$ sodium citrate buffer ( $\mathrm{pH}$ 6.1) for 3 min. After blocking of endogenous peroxide activity using $0.03 \%$ hydrogen peroxide, the sections were incubated for $2 \mathrm{~h}$ with a primary antibody (1:1 to 1:2 diluted culture soup for mAbs, 1:1,000 for polyclonal antibody) against CCDC110 at room temperature. The samples were washed and then incubated with HRP-conjugated anti-mouse IgG (Dako EnVision+system-HRP [DAB], Dako, Carpinteria, CA, USA) for $20 \mathrm{~min}$ at room temperature. After washing, the chromogen was developed for $2 \mathrm{~min}$. The tissue sections were then counterstained with weak hematoxylin. The images of IHC was obtained using Olympus BX51 upright microscope (Olympus, Tokyo, Japan) equipped with digital camera.

\section{RESULTS}

\section{The specificity of mAbs determined by immunoblotting and immunoprecipitation}

With ELISA screening, nine hybridoma clones reactive with CCDC110 protein were obtained. The isotypes of each CCDC110 mAbs were tested and determined (Table 1). The reactivity of all $\mathrm{mAbs}$ against both endogenous and overexpressed CCDC110 proteins was tested (Fig. 1A). As shown in Fig. 1A, each clone of the mAbs readily detects overexpressed recombinant proteins. However, the detection of endogenous CCDC110 protein around $105 \mathrm{kDa}$ seems to be elusive.

Table 1. Characteristics of CCDC110 mAbs

\begin{tabular}{cccc}
\hline \hline mAb & Clone name & Isotype & Immunoblotting reactivity against \\
\hline M1 & 5A10C12G3 & $\operatorname{lgG} 1$ & $\mathrm{E}( \pm), \mathrm{O}(+++)$ \\
M2 & 4D9A12D4 & $\operatorname{lgG} 2 \mathrm{~b}$ & ${ }^{*} \mathrm{E}(+), \mathrm{O}(+++)$ \\
M3 & 4D9A12B10 & $\operatorname{lgG} 2 \mathrm{~b}$ & $\mathrm{E}(+), \mathrm{O}(+++)$ \\
M5 & 1A6H10 & $\operatorname{lgG} 1 / \operatorname{lgG} 2 \mathrm{~b}$ & $\mathrm{E}( \pm), \mathrm{O}(+++) \operatorname{IP}(++)^{*}$ \\
M6 & $3 \mathrm{~A} 1 \mathrm{E} 12$ & $\operatorname{lgG} 1$ & $\mathrm{E}( \pm), \mathrm{O}(+++)$ \\
M7 & $2 \mathrm{H} 11 \mathrm{H} 7$ & $\operatorname{lgG} 1$ & $\mathrm{E}( \pm), \mathrm{O}(++) \mathrm{IP}(+)^{*+}$ \\
M8 & $\mathrm{A}-4-3$ & $\operatorname{lgG} 1$ & ${ }^{*} \mathrm{E}(+), \mathrm{O}(+++)$ \\
M11 & $\mathrm{B}-10$ & $\operatorname{lgG} 1$ & ${ }^{*} \mathrm{E}(+), \mathrm{O}(+++)$ \\
M12 & $\mathrm{C}-12-12$ & $\operatorname{lgG} 1$ & ${ }^{*} \mathrm{E}(+), \mathrm{O}(+++)$ \\
\hline
\end{tabular}

The results of isotyping, specificity and sensitivity of Immunoblotting, and the efficacy of immunoprecipitation was presented. $\mathrm{E}$, untransfected cell lysate; OE, detection of ectopically overexpressed protein; IP immunoprecipitation. The immunoprecipitation efficacy of M5 and M7 mAbs were assessed.

The relative immunoblotting reactivity is divided into four levels:,,, \pm++++++ .

* M5 clone can immunoprecipitate both endogenous and ectopically overexpressed protein.

* M7 clone immunoprecipitate ectopically overexpressed protein.

* The detection of endogenous protein was assessed only in immunoprecipitated samples.

CCDC110, coiled-coil domain containing 110; E, endogenous; O, overexpressed; IP, immunoprecipitated. 
A

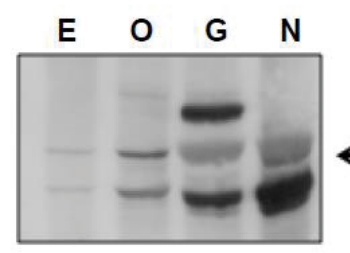

M2

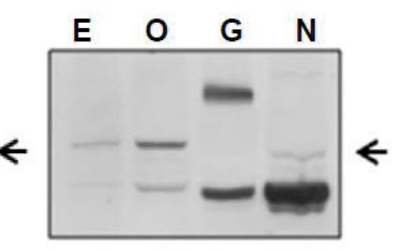

M3

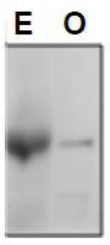

M5

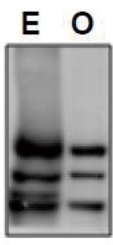

M6

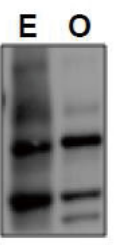

M7

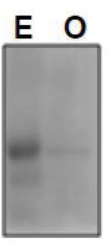

M8

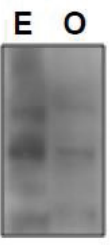

M11

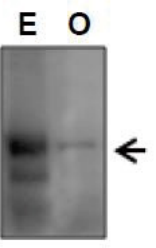

M12
B

IP

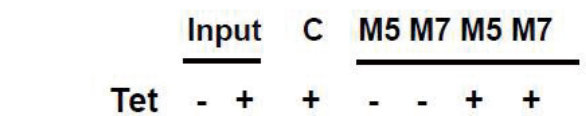

D

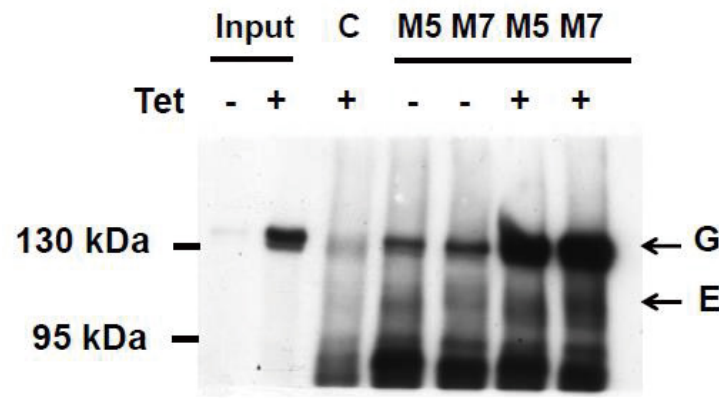

F

IP

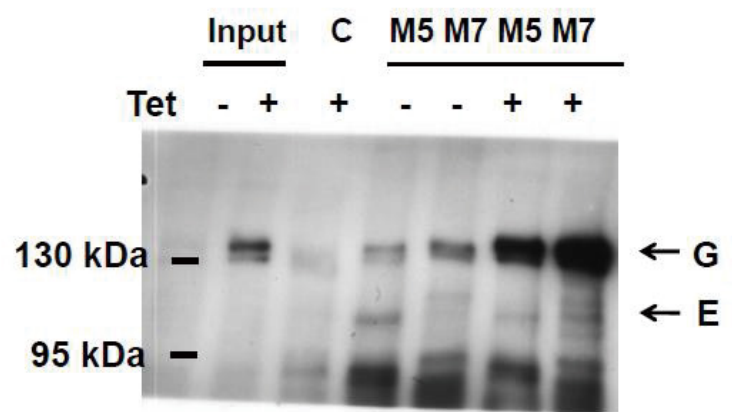

IP

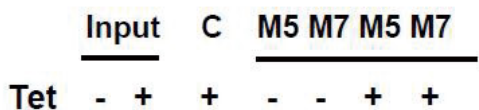

E

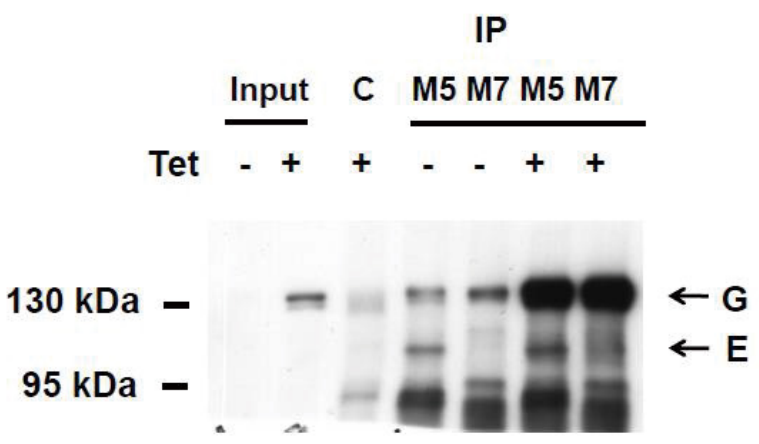

G

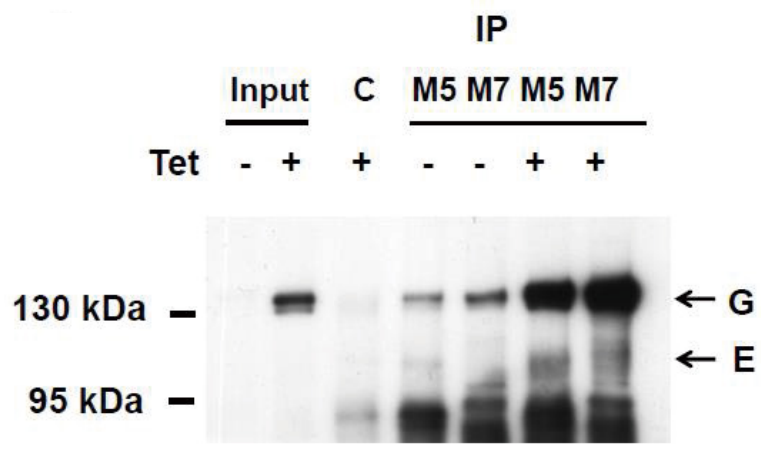

Fig. 1. Characterization of CCDC110 mAbs. (A) Cell lysates from U2-OS cells, transfected with indicated expression constructs (O, pCl neo CCDC110; G, pEGFPC1 CCDC110; N, pEGFPC1 CCDC110(1-527)), were electrophoresed and immunoblotted with indicated mAbs. The diluted hybridomal culture soup $(1: 10)$ was used as primary Ab. E: endogenous. The arrows indicate the native CCDC110 protein. (B-G) Immunoprecipitation of recombinant EGFP-CCDC110 protein from the cell lysates of U2-OS cells containing the tetracycline inducible expression vector for EGFP-CCDC110 protein. The cell lysates were prepared either in the absence $(-)$ or presence $(+)$ of $1 \mu \mathrm{g} / \mathrm{mL}$ doxycycline. After immunoprecipitation with indicated Abs, immunoblot was performed with M2 (B), M3 (C), M5 (D), M8 (E), M11 (F), and M12 (G) Abs. Arrows with G indicate EGFP-CCDC110 and arrows with E indicate endogenous CCDC110. CCDC110, coiled-coil domain containing 110; EGFP, enhanced green fluorescence protein. 
To test whether mAbs can immunoprecipitate CCDC110 protein, we used Tet-inducible U2OS cells which can produce EGFP-CCDC110 protein upon treatment with tetracycline or their related delivatives. The proteins obtained from the lysates of both doxycycline-treated and untreated cells were immunoprecipitated with hybridoma mAbs and blotted with obtained mAbs (Fig. 1BG). M5 Ab reacted with both endogenous and overexpressed CCDC110. However, M7 clone can specifically immunoprecipitate the overexpressed CCDC110 but not the endogenous CCDC10. The immunoprecipitated endogenous proteins can be detected by M2, M3, M5, M8, and M11 Abs which can also detect overexpressed CCDC110. Although the immunoprecipitated endogenous proteins could be detected in the IP sample, the corresponding signals of the protein in cell lysate (input) were seldom detected. The characteristics of each clone are summarized in Table 1.

\section{Detection of recombinant EGFR-CCDC110 protein by $\mathrm{mAbs}$ in immunofluorescence staining}

To test whether the CCDC110 proteins can be detected by mAbs, U2-OS cells were transfected with pShuttle CMV EGFP MGC full, which can express CCDC110 fused with EGFP, and immunofluorescence staining was conducted. As shown in Fig. 2, hybridoma clones M5, M8, and M11 can detect ectopically overexpressed EGFP-CCDC110 protein. The green fluorescence signal of EGFP colocalized with the red fluorescence signal of each mAbs stained with Alexa 532 conjugated anti-mouse IgG. These mAbs were further tested in IHC of tissue sections.

\section{IHC reveals CCDC110 is prominent in testicular tubule area}

Previously we raised polyclonal antisera and purified it with GST-CCDC110 affinity purification (Park et al., 2007). We tested anti-CCDC110 mAbs for IHC of paraffin-embedded

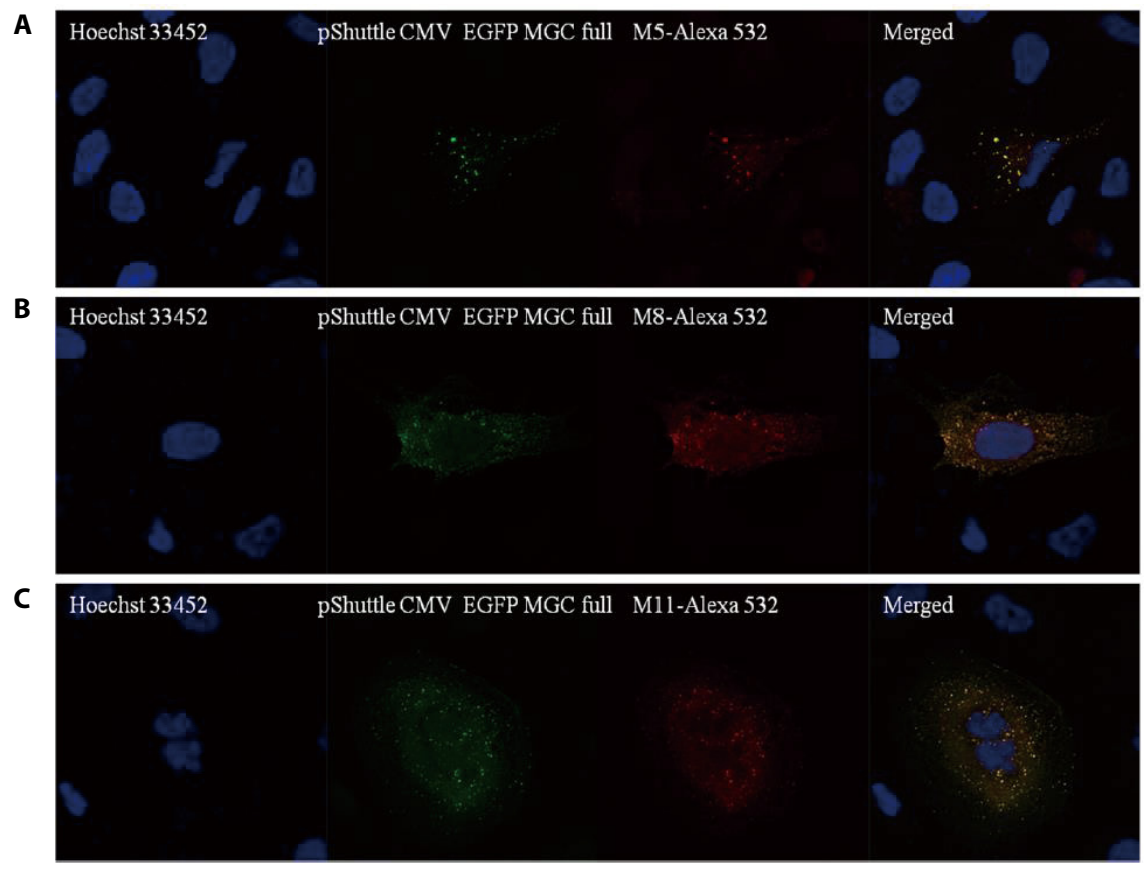

Fig. 2. EGFP-CCDC110 can be detected by mAbs in immunofluorescence staining. U2-OS cells, expressing EGFP-CCDC110 from pShuttle CMV EGFP MGC full, were stained with mAbs M5-Alexa 535 (A), M8-Alexa 532 (B), M11-Alexa 532 (C), respectively. EGFP, enhanced green fluorescence protein; CCDC110, coiled-coil domain containing 110 . 


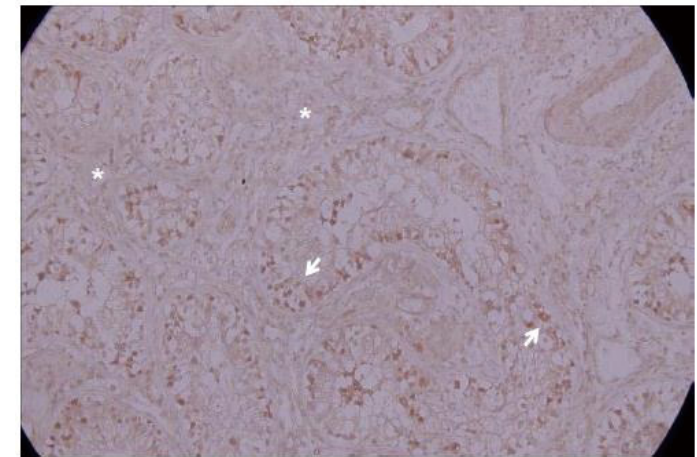

P5A 200X

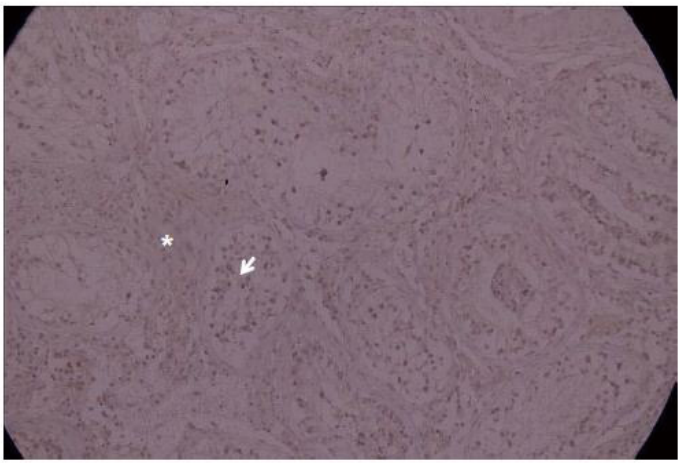

M5 200X

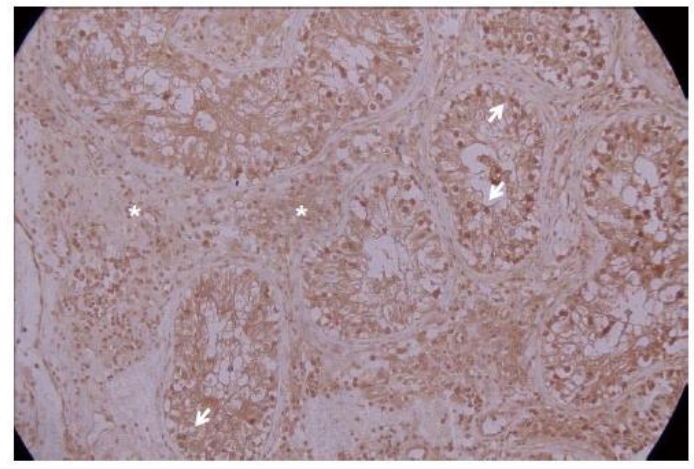

M7 200X

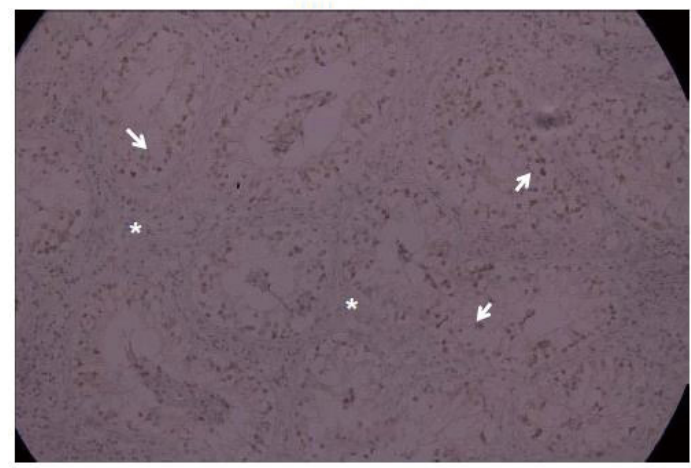

M11 200X

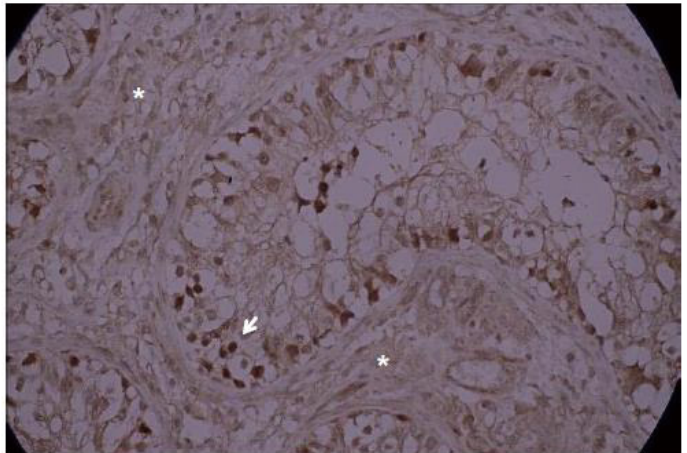

P5A 400X

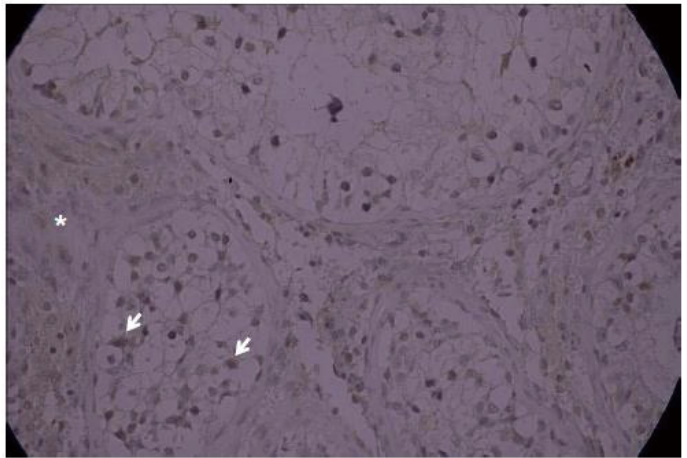

M5 400X

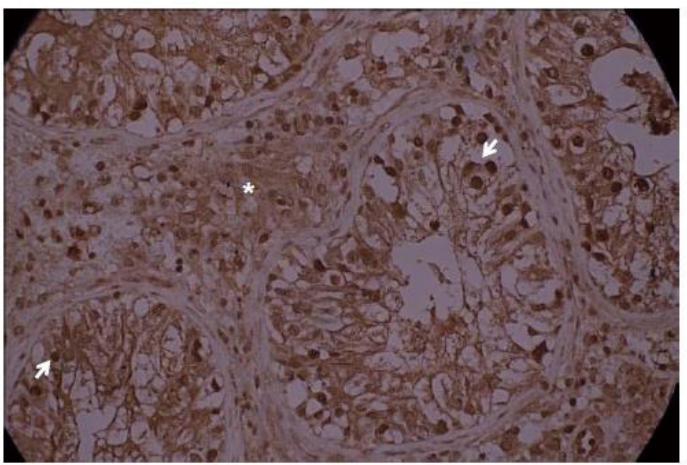

M7 400X

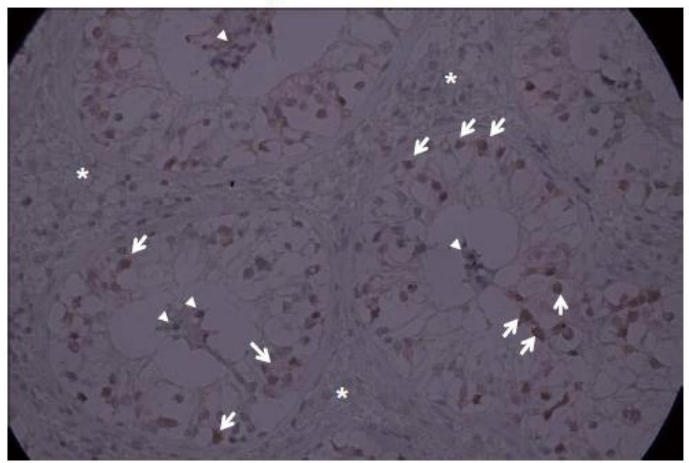

M11 400X

Fig. 3. CCDC110 protein expression in testis. Human testis tissue sections were tested with affinity-purified polyclonal Ab (P5A), mAbs M5, M7, or M11, respectively. The 200X and 400X indicate microscopic magnification fold. Arrows indicate positively stained spermatogenic cells in basal area of seminiferous tubule. Arrowheads indicate weakly stained spermatocytes in lumen. Asterisks indicate stromal connective tissue area which have negative staining. CCDC110, coiled-coil domain containing 110. 
testis block and compared with affinity-purified polyclonal Ab (Fig. 3). Polyclonal Ab showed a strong signal in the seminiferous tubule area, whereas no signal was detected in the stromal region. The IHC using M7 antibody showed high background signal in the extra-tubule area. In 200x magnification, the M5 and M11 mAbs share a similar IHC staining pattern: prominent in the tubular basal area and weak in the stromal area, which is similar to affinity-purified polyclonal $\mathrm{Ab}(\mathrm{P} 5 \mathrm{~A})$. The IHC of M11 mAb shows a more significant difference between parenchymal spermatogenic cells and stromal cells, observed both in 200x and 400x magnification. The 400x magnification of M11 IHC reveals both nuclear and cytoplasmic staining in spermatogenic cells in the basal region of tubule abundant of spermatogonia. As the cells in the seminiferous tubule area have high replication potential due to spermatogenesis compared with those in the stromal region in which connective tissues and fibroblasts exist, the $\mathrm{Ab}$ seems to detect highly replicative spermatogenic cells.

\section{Ectopic overexpression of EGFP-CCDC110 results in delayed reentry into G2/M phase}

The Tet-On U2-OS cells carrying inducible EGFP-CCDC110 protein were synchronized with mitotic phase arrest by nocodazole treatment followed by mitotic exit and entry into G1 phase by shake-off plating. The levels of cell cycle markers were evaluated (Fig. 4). As expected, the nocodazole arrested cells showed highest levels of cyclin B. The signals of cyclin B reappear at $9 \mathrm{~h}$ and show peaks at 21-27 h after $1^{\text {st }}$ mitotic exit in doxycycline untreated control cells. However, the increase of cyclin B in the Tet-induced EGFP-CCDC110 expressing cells was delayed and suppressed. The increase of cyclin B in EGFP-CCDC110-overexpressed cells coincided with the gradual decrease of EGFP-CCDC110. The expression of cyclin A also shows a delayed increase, similar to cyclin B. The level of induced EGFP-CCDC110 is disappeared in nocodazole treated $\mathrm{M}$ phase-arrested cell and increases at $6 \mathrm{~h}$, peaked at $12 \mathrm{~h}$ and decreases gradually. The level of p21 was increased in EGFP-CCDC110-induced cells compared to uninduced cells. The immunoblot signals of endogenous CCDC110 was too weak to detect under these conditions.

\section{DISCUSSION}

CCDC110 is one of the members of CTAs and its expression is minimal in normal tissues but is increased in testis tissues and several types of cancers. Testicular tissues show the characteristic high activity of cell proliferation due to spermatogenesis in the seminiferous area and the proteins specific to testicular tissues are generally not exposed to self-immune defense system (Chen et al., 1997). CTAs are known to be a the valuable druggable candidate for immunotherapy or cancers (Chen et al., 1997; Scanlan et al., 2002; Scanlan et al., 2004). In addition, CCDC110 contains C-terminal CCD and ZIP repeats at amino acid residues 448-483. It also contains NLS motif at 338-355 and SMC motif at 431-790 (Park et al., 2007). The ZIP motif is important for protein oligomerization and CCDC110 has five repeats of ZIP exceeding the minimal requirement number of repeats. Several microarray results showed that the CCDC110 protein increases in cells or conditions where cell proliferation is active. However, little is known about cellular function except for characteristic overexpression of mRNA levels in various cancer tissues such as tongue cancer, melanoma, and liver cancer. A recent study showed that CCDC110 auto-antibodies are detected in the cohort of gastric cancer patients (Hoshino et al., 2017).

In this work, we developed an efficient CCDC110 mAb because the production of $\mathrm{mAb}$ against CTAs is useful for its clinical application as cancer markers in pathology, serological diagnosis and prediction of cancer prognosis. All the obtained $\mathrm{mAbs}$ showed stronger reactivity in 
Doxycycline (+)
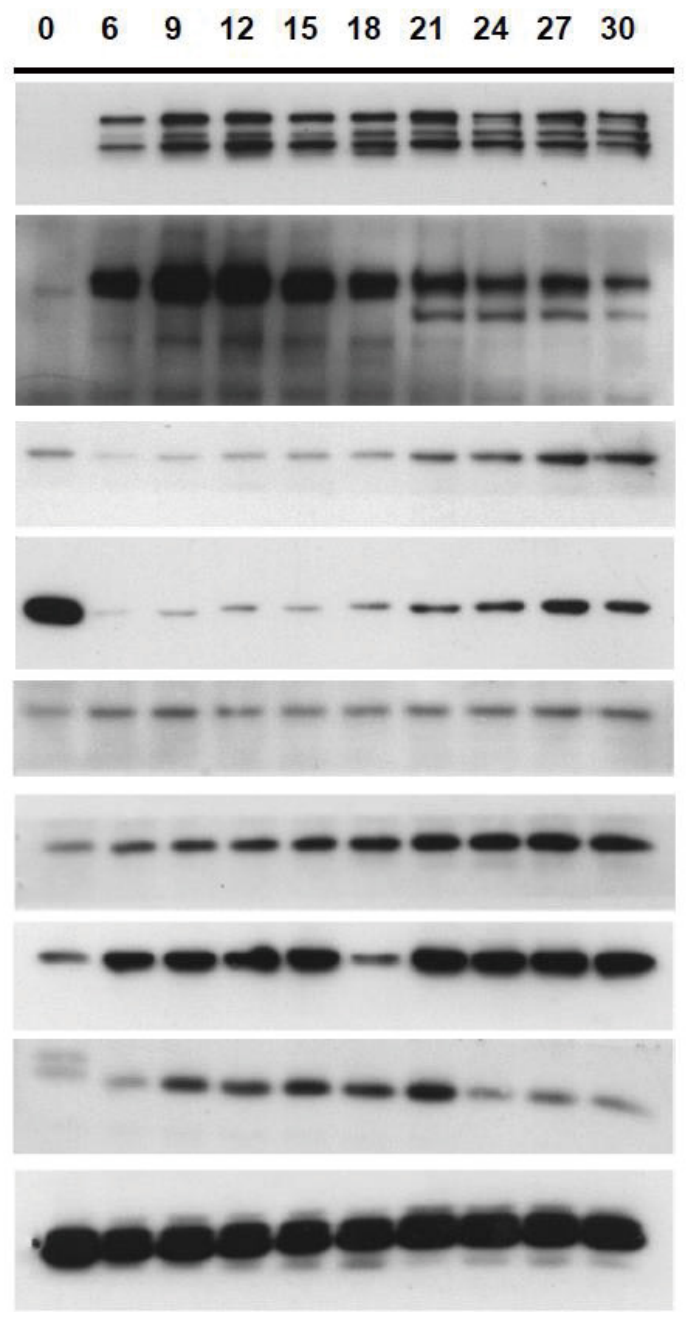

Doxycycline (-)
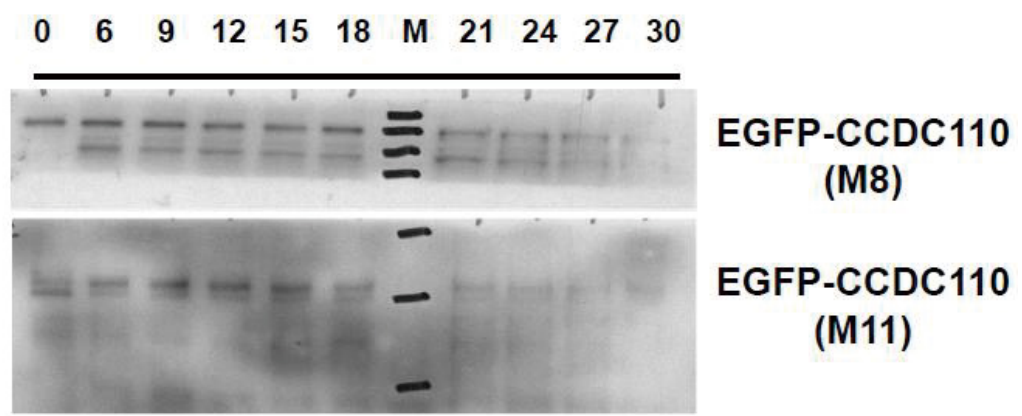

EGFP-CCDC110

(M11)

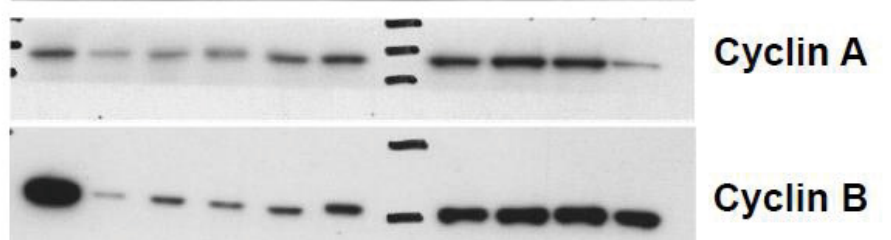

\section{Cyclin D1}

Cyclin D3

p53

p21-waf1

ERK

Fig. 4. The overexpressed CCDC110 is controlled according to cell cycle and delays cell cycle progression. The Tet-On U2-OS cells carrying inducible EGFP-CCDC110 gene were treated with tetracycline $(200 \mathrm{ng} / \mathrm{mL})$ and nocodazole $(200 \mathrm{ng} / \mathrm{mL})$ for $18 \mathrm{~h}$, then the floating cells representing mitotic arrest were harvested by shake-off and plated to the nocodazole-free and either doxycycline containing or free medium. The cells were harvested at indicated time points and the lysates were immunoblotted with antibodies for indicated proteins. The ERK protein was used as the loading control. CCDC110, coiled-coil domain containing 110; EGFP, enhanced green fluorescence protein.

immunofluorescence staining of exogenously overexpressed proteins. The immunohistochemical analysis of mAb M11 revealed similar or better outcome with affinity-purified polyclonal antibody. However, we did not compare the efficacy of our developed antibodies with the commercially available antibodies. The M11 mAb was strongly stained in cells in the basal layer of tubules rich in spermatogenic cells and negatively stained in stromal connective tissue regions. The M11 stained signal exists both in the cytoplasm and nucleus, showing that the M11 is suitable for application of IHC targeting CCDC110 in various cancer tissues. Thus, we believe that this $\mathrm{mAb}$ will be useful for characterizing the expression levels of $\mathrm{CCDC} 110$ and its functional role in various cancer tissues.

Notably, we found that the levels of overexpressed CCDC110 inversely correlated with the levels of cyclin B in TRE harboring Tet-On U2-OS cells, suggesting that this protein is under the control of certain protein degradation system activated in the G2/M phase. However, we cannot 
rule out the possibility that this result is caused by potential artifacts associated with lentiviral infection in the engineered cell line used in our study. Nevertheless, our results suggest that CCDC110 can be regulated during cell cycle progression and its persistent expression can impede cell cycle progression. However, it is also plausible that the degradation of overexpressed CCDC110 is mediated by dysregulated protein process under our ectopic expression condition. Further study is needed to elucidate a more detailed molecular mechanism associated with cell cycle-controlled degradation of CCDC110.

\section{REFERENCES}

Boulikas T (1993) Nuclear localization signals (NLS). Crit Rev Eukaryot Gene Expr 3:193-227.

Chen YT, Scanlan MJ, Sahin U, Türeci Ö, Gure AO, Tsang S, Williamson B, Stockert E, Pfreundschuh M, Old LJ (1997) A testicular antigen aberrantly expressed in human cancers detected by autologous antibody screening. Proc Natl Acad Sci USA 94:1914-1918.

Cobbe N, Heck MMS (2000) Review: SMCs in the world of chromosome biology- from prokaryotes to higher eukaryotes. J Struct Biol 129:123-143.

Condomines M, Hose D, Raynaud P, Hundemer M, De Vos J, Baudard M, Moehler T, Pantesco V, Moos M, Schved JF, Rossi JF, Rème T, Goldschmidt H, Klein B (2007) Cancer/testis genes in multiple myeloma: Expression patterns and prognosis value determined by microarray analysis. J Immunol 178:3307-3315.

Harlow E, Lane D (1988) Antibodies: A Laboratory Manual. Cold Spring Harbor Laboratory Press, New York, NY.

Hoshino I, Nagata M, Takiguchi N, Nabeya Y, Ikeda A, Yokoi S, Kuwajima A, Tagawa M, Matsushita K, Satoshi Y, Hideaki S (2017) Panel of autoantibodies against multiple tumorassociated antigens for detecting gastric cancer. Cancer Sci 108:308-315.

Landschulz WH, Johnson PF, McKnight SL (1988) The leucine zipper: A hypothetical structure common to a new class of DNA binding proteins. Science 240:1759-1764.

Lee KH (2012) Ectopic expression of cenexin1 S796A mutant in ODF2+/- knockout background causes a sperm tail development defect. Dev Reprod 16:363-370.

Lucerna M, Pomyje J, Mechtcheriakova D, Kadl A, Gruber F, Bilban M, Sobanov Y, Schabbauer G, Breuss J, Wagner O, Bischoff M, Clauss M, Binder BR, Hofer E (2006) Sustained expression of early growth response protein-1 blocks angiogenesis and tumor growth. Cancer Res 66:6708-6713.

Monji M, Nakatsura T, Senju S, Yoshitake Y, Sawatsubashi M, Shinohara M, Kageshita T, Ono T, Inokuchi A, Nishimura Y (2004) Identification of a novel human cancer/testis antigen, KMHN-1, recognized by cellular and humoral immune responses. Clin Cancer Res 10:6047-6057.

Namekawa SH, Park PJ, Zhang LF, Shima JE, McCarrey JR, Griswold MD, Lee JT (2006) Postmeiotic sex chromatin in the male germline of mice. Curr Biol 16:660-667.

Otu HH, Naxerova K, Ho K, Can H, Nesbitt N, Libermann TA, Karp SJ (2007) Restoration of liver mass after injury requires proliferative and not embryonic transcriptional patterns. J Biol Chem 282:11197-11204.

Park HJ, Seo HJ, Kim HW, Kim JS, Hwang SY, Seong YS (2007) The centrosomal localization of KM-HN-1 (MGC33607) depends on the leucine zipper motif and the C-terminal coiled-coil domain. Exp Mol Med 39:828-838.

Scanlan MJ, Gure AO, Jungbluth AA, Old LJ, Chen YT (2002) Cancer/testis antigens: An expanding family of targets for cancer immunotherapy. Immunol Rev 188:22-32. 
Scanlan MJ, Simpson AJG, Old LJ (2004) The cancer/testis genes: Review, standardization, and commentary. Cancer Immun 4:1.

Soung NK, Kang YH, Kim K, Kamijo K, Yoon H, Seong YS, Kuo YL, Miki T, Kim SR, Kuriyama R, Giam CZ, Ahn CH, Lee KS (2006) Requirement of hCenexin for proper mitotic functions of polo-like kinase 1 at the centrosomes. Mol Cell Biol 26:8316-8335.

Vaughan HA, St Clair F, Scanlan MJ, Chen YT, Maraskovsky E, Sizeland A, Old LJ, Cebon J (2004) The humoral immune response to head and neck cancer antigens as defined by the serological analysis of tumor antigens by recombinant cDNA expression cloning. Cancer Immun 4:5. 
